\title{
Surgery Path Planning for Lung Biopsy Based on Pareto Optimization
}

\author{
Nan Bao*, Jingyi Jia, Yueyao Chen,Zhizhou Li, Chongchong Song, Ruotong Zhao \\ Northeastern Univeristy, College of Medicine and Biological Information Engineering, Shenyang, \\ China \\ baonan@bmie.neu.edu.cn
}

Keywords: Surgery path planning, Lung biopsy, CT image-guided surgery, Pareto optimization

\begin{abstract}
CT image-guided lung biopsy surgery is the gold standard for lung tumor diagnosis. The preoperative path planning of the surgery is necessary, which aims to find some optimal needle insertion points on the patient's skin. The traditional path planning method is that the surgeons estimate the puncture paths by observing the patient's CT images, which is highly dependent on the experience of surgeons and affects the success rate of the surgery due to improper path selection. In this work, we proposed an intelligent lung biopsy surgery path planning method, which solved a multi-objective optimization problem. We segmented some chest important organs based on CT images, and quantitatively analyzed multiple clinical criteria. The concept of Pareto optimization was introduced to solve the multi-objective optimization problem in the method. Finally, some optimal surgery paths were provided for the surgeons. The retrospective evaluation of clinical data was performed, which was proved that the proposed method could be accepted in lung biopsy surgery.
\end{abstract}

\section{Introduction}

As one of common malignant tumors, lung cancer has the highest morbidityandmortality [1]. CT image-guided lung biopsy is the main approach to diagnose lung cancer ([2], [3]). Lung biopsy requires inserting the needle into the skin to obtain a small amount of lung tissue for analysis [4]. Because there are many important organs around the lung, and a plenty of clinical criteria have to be considered in lung biopsy surgery, it is of great significance to carry out preoperative surgery path planning. The traditional method for clinicians to plan a surgery path highly depends on their experiences, and they usually observe the patient's CT images and design the surgery paths manually. If the planning path is not reasonable, especially for young surgeon, the success rate of the lung biopsy surgery would be affected. Therefore, it is necessary to design an intelligent surgery path planning method. Based on the preoperative CT images of patients, several optimal surgery paths can be designed automatically and provided for surgeons, which could improve the surgery success rate and reduce the burden on surgeons [5].

Several related methods have been proposed in the past few years. However, most of them focus on the brain and abdominal surgery ([6], [7]), and few of them research on the lung biopsy surgery path planning. Furthermore, in these methods, the multi-objective optimization problem is usually solved based on the weight optimization ([8], [9]). They have to select some of all clinical criteria and give them higher weight values based on the experience, which is always dominated by the subjectivity and diversity. These methods have higher requirements for surgeons' clinical experience, so it is difficult to ensure the accuracy and objectivity of the surgery path planning. 
In this paper, we propose an intelligent multi-objective surgery path planning method based on the clinical criteria of lung biopsy. In order to avoid the distortion caused by the man-made weight, we introduce Pareto optimization method to calculate the optimal paths. Firstly, all the important chest organs around the surgery paths are segmented. Then the surgical criteria are quantitatively analyzed based on the segmentation results. Finally, the optimal surgery paths are calculated based on Pareto optimization method, which does not need surgeons to set the weights artificially. The proposed method considers each clinical criterion synthetically, and obtains several optimal paths for surgeons, which are more accurate and robust.

This paper is organized as follows: First, we segment chest organs, quantify the surgical criteria and utilize the concept of Pareto optimization to find the optimal surgery path in the process of lung biopsy surgery (section 2); second, we compare the obtained paths with that of the surgeons for evaluation (section 3); finally, the conclusions of the study are shown in section 4.

\section{Methods}

\subsection{Chest Organs Segmentation Based on CT Images}

According to the anatomical structure of human chest and the clinical requirements, we need to segment some important organs based on chest CT images for lung biopsy surgery path planning, including skin, vertebrae and ribs, trachea and bronchus, pulmonary vessels, mediastinumand interlobar fissures.

In this paper, we comprehensively considered the features of all the organs segmented, and designed the segmentation strategy. After pre-processing and denoising the CT images, the chest area was segmented and the skin area was obtained by morphological operation. Then, in the chest area, the trachea and bronchus, vertebrae and ribs, and lung parenchyma were segmented. Based on these results, the structures of the pulmonary blood vessels and mediastinum were identified. Finally, the pulmonary lobes and interlobar fissures were extracted based on the classification of bronchus and blood vessels in the lung parenchyma. Using the strategy, all chest organs are segmented fast, and the results meet the clinical requirements.

\subsection{Quantitative Analysis of Clinical Criteria for Lung Biopsy}

Lung biopsy surgery has its unique clinical criteria, including three clinical constraints and six objectives. In order to make the surgery path much safer and calculate the optimal surgery paths, these clinical criteria have to be analyzed quantitatively.

There are three clinical constraints: (1) Chest Important Organs Avoidance Constraint: the surgery path and its extension line cannot cross the important organs including the vertebrae and ribs, the interlobar fissures and the mediastinum area; (2) Puncture Needle Length Limit Constraint: the length of the surgery path has to be within the effective working range which is less than $21 \mathrm{~cm}$.; (3)Insertion Angle Constraint: the puncture needle should not be too inclined during the lung biopsy surgery. In order to improve the success rate, the insertion angles between the surgery path and the two surfaces should set to be greater than $20^{\circ}$.

There are six clinical objectives: (1) Surgery Path Length Sub-objective: the total length of the surgery path and the length of the surgery path in the lung parenchyma are needed to be shorter. (2) Surgery Path Distance Sub-objective: the distance between the chest important organ and the surgery path, and the distance between the chest important organ and the extension line of the 
surgery path are needed to be farther.(3)Path Insertion Angle Sub-objective: the insertion angle between the surgery paths and the skin, and the angle between the surgery paths and the surface of the lung parenchyma are needed to be closer to90 .

\subsection{Optimal Surgery Path Calculation with Multiple Objectives}

In this paper, based on the quantitative analysis results of the clinical criteria, the surgery path optimization method with multiple constraints and sub-objectives is proposed. The path optimization aims to minimize the surgery risk under the premise of obeying the three constraints, and find some optimal paths for the six sub-objectives. Generally, all of the sub-objectives in multi-objective optimization problem are constrained by decision variables. It is impossible to achieve the optimal values of all sub-objectives simultaneously, and some compromise solutions have to be found. Pareto optimization method is introduced to solve the optimal path calculation of the lung biopsy surgery. The six sub-objectives of surgery path risk are used as the six sub-objective functions of Pareto optimization, which can optimize and evaluate the paths without setting man-made risk weights. Pareto optimization was first introduced in econometrics [10].

First of all, we removed all candidate surgery paths which did not obey the three constraints including chest important organs avoidance constraint, puncture needle length limit constraint and insertion angle constraint. The remaining paths were effective in the lung biopsy surgery. Then, a Pareto coordinate system was established with each two sub-objectives, and the effective paths were projected on it. For instance, we projected the insertion points of all effective paths into the Pareto coordinate system in blue dots shown as Figure 1, whose horizontal axis is the risk function value of the skin insertion angle objective, and the vertical axis is the risk function value of the path length objective. According to the definition of the Pareto optimization, we calculated and obtained $\mathrm{P}_{1}, \mathrm{P}_{2}, \mathrm{P}_{3}, \mathrm{P}_{4}, \mathrm{P}_{5}$ as the set of Pareto Front, which were the compromise solutions of the two sub-objectives. The paths represented by the five points are the optimal paths under the two sub-objectives.

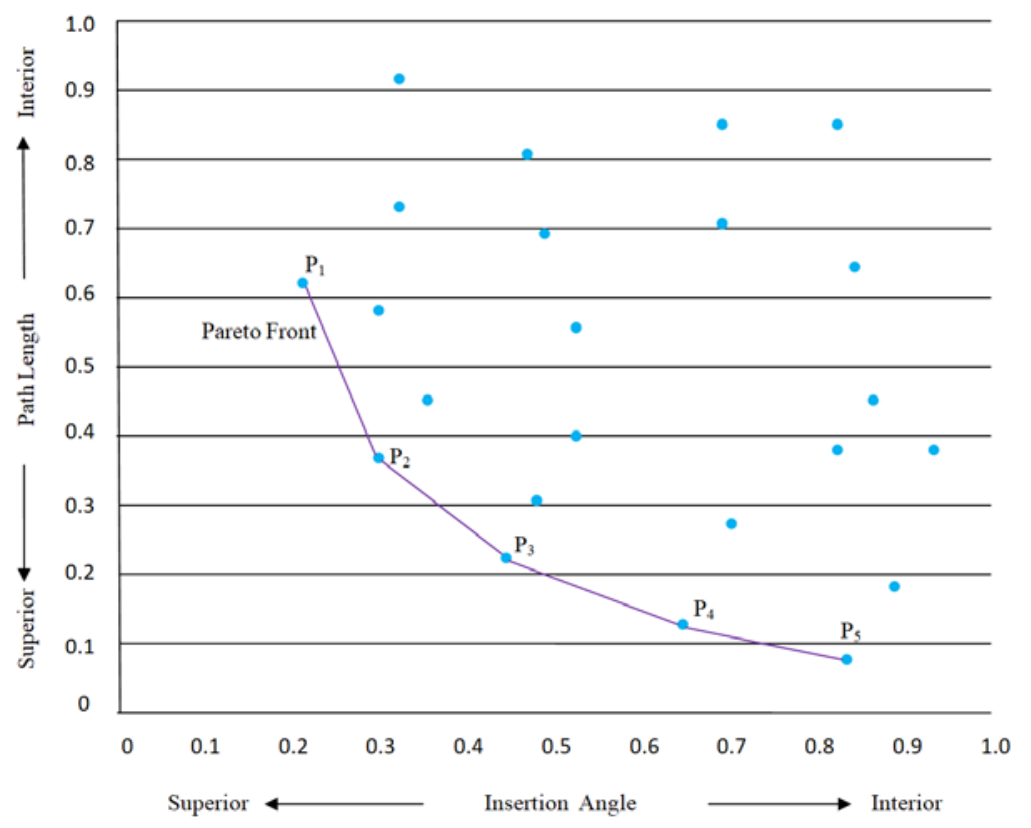

Figure 1. Pareto coordinate system of the path length and insertion angle sub-objectives

The above procedure was repeated on the other sub-objectives until we got 15 sets of Pareto 
Front. Then, they are intersected to obtain the final insertion points considered as the optimal surgery paths.

\section{Results}

\subsection{Experimental Evaluation Method}

This paper uses retrospective evaluation method to verify the path optimization method, involving six sets of patient's chest CT data ( $1 \mathrm{~mm}$ slice thickness, $512 \times 512$ in-plane resolution) which was scanned before and after the successful lung biopsy surgery. The effective insertion areas of the skin are found by the following steps. Firstly, all important organs were segmented in the preoperative CT images, and the skin insertion points of the surgery paths disobeying the three constraints were removed.

Then, the path planning method was performed, and we obtained some optimal surgery paths. We registered the preoperative and postoperative CT images, and projected the surgeon's clinical paths using in the lung biopsy surgery on the postoperative CT images to the preoperative CT images. The clinical paths and the optimal paths calculated by our proposed method are all marked. Two experienced lung biopsy surgeons evaluated these surgery paths without knowing the truth to avoid the impact of subjective factors.

\subsection{Comparison with Clinical Path and Calculated Paths}

We compared the clinical path and calculated paths in the six sub-objectives shown in Figure 2. The top of each histogram is the corresponding sub-objective function values of the effective paths with the lowest risk, which is represented by green; the bottom red area is the corresponding sub-objective function values of the effective paths with the highest risk. So, in the histogram of each sub-objective, the path risk increases from top to bottom. The surgery risk sub-objective function value of clinical path is shown as a black horizontal line in the six sub-objective histograms.

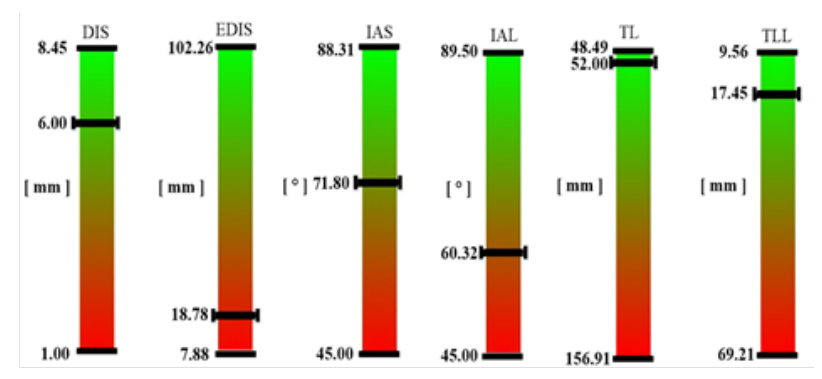

Figure 2. The risk comparison on the six sub-objectives

It can be seen that the clinical path was not all optimal with regard to six sub-objectives, and even performed poorly in some objectives. Among them, the clinical path has a better score in terms of the sub-objectives of the total length and the length in the lung. It is indicated that surgeons pay more attention to the length of the path, and the path lengths are easier to be observed in the CT images. Meanwhile, the scores of the other four sub-objectives are relatively poor. Because surgeons planned the surgery paths by observing the two-dimensional slicers of the CT image, it is difficult to consider all sub-objectives simultaneously. Therefore, it is necessary to study the intelligent surgery path planning method. 


\subsection{Comparison with Clinical Path and Calculate Paths in Pareto Coordinate System}

The Pareto coordinate systems were established based on the sixsub-objective functions. The insertion points of the clinical path and all effective paths are projected in the Pareto coordinate system. For instance, threePareto coordinate systems using one set of patient's CT image data are shown in Figure 3. The effective paths are represented by blue dots, and the clinical paths are represented by red dots. We can see that the insertion point of the clinical path is not on Pareto Front in the three coordinate systems, so it can be further optimized.

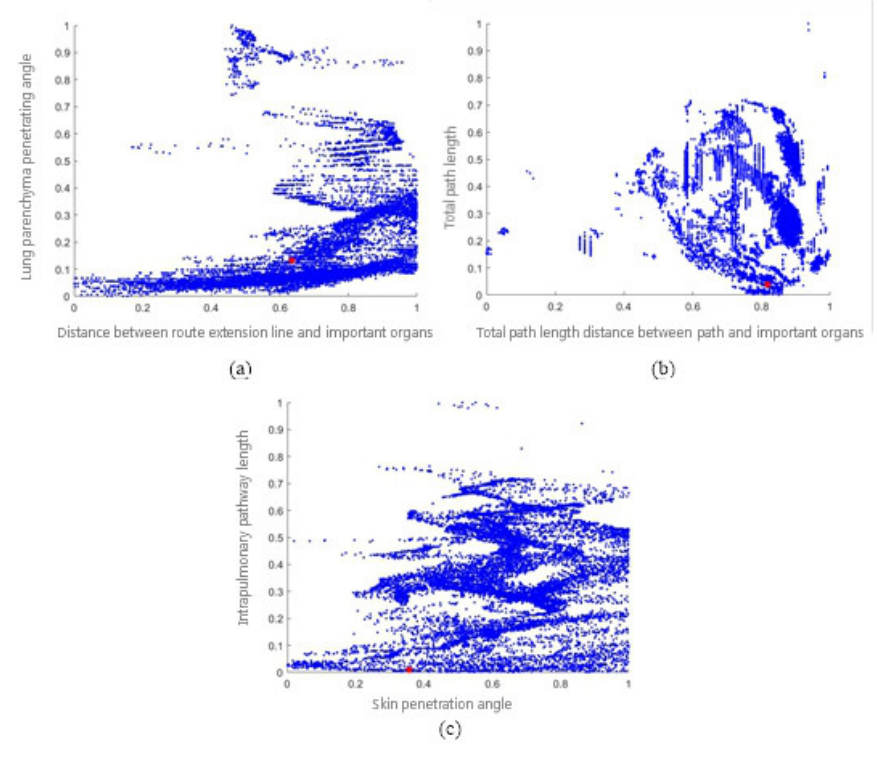

Figure 3. The risk comparison in three Pareto coordinate systems

\subsection{Comparison with Clinical Path and Calculate Paths by Surgeons}

The calculated paths and the clinical paths were displayed on the preoperative CT images, which were evaluated by two clinicians who have many years of experiences in the lung puncture surgery. The results of clinicians' evaluation showed that all the optimal paths met surgery requirements. Moreover, the calculated paths performed better than $75 \%$ when the clinician selected the best optimal path, and they are consistent with $17 \%$ of the clinical paths.

\section{Conclusions}

Preoperative path planning is the basis of a successful surgery. In this paper, we have proposed an intelligent optimal path planning method in the lung biopsy surgery based on the quantitative analysis of the clinical criterion. The Pareto optimization has been introduced to solve the multi-objective problem without setting man-made weights to obtain the optimal surgery paths for the surgeons. The retrospective evaluation results have shown that the optimal paths calculated based on the proposed method meet the requirements of the surgery, and most of their performance are equal to or even better than the clinical paths used in surgery. In future, we will use more clinical data to verify and improve our proposed method.

\section{Acknowledgements}

The authors would like to thank the editor and reviewers for improving the quality of the paper. This work was partially supported by National Natural Science Foundation of China (NSFC) [Grant 
No. 61701103], the Fundamental Research Funds for the Central Universities [Grant No.N181904002], and the National Training Program of Innovation and Entrepreneurship for Undergraduates [Grant No.201910145100].

\section{References}

[1]Shelley M. World Cancer Report 2014. Geneva, Switzerland: World Health Organization, International Agency for Research on Cancer, WHO Press, 2015. Advances in Nutrition, 7(2):418-419, 2016 .

[2]Laspas F, Roussakis A, Efthimiadou R, et al. Percutaneous CT-guided fine-needle aspiration ofpulmonary lesions: Results and complications in 409 patients. Journal of Medical Imaging \& Radiation Oncology,52(5): 458, 2008.

[3]Taleb S, Jalaeian H, Frank N, et al. Is a Routine Chest X-ray Necessary in Every Patient After Percutaneous CT-Guided Lung Biopsy? A Retrospective Review of 278 Cases. CardiovascInterventRadiol, (1): 1-6, 2017.

[4]https://www.healthline.com/health/lung-needle-biopsy\#risks

[5]Arnolli MM, Hanuman NC, Franken M, et al. An overview of systems for CT- and MRI-guided percutaneous needle placement in the thorax and abdomen. J Med Robotics Comput Assist Surg , 11: 458-475, 2015.

[6]Hamze N , Collet P , Essert C . Evolutionary approaches for surgical path planning: a quantitative study on Deep Brain Stimulation. Evolutionary ComputationIEEE, 2017.

[7]Seitel A, Engel M, Sommer CM, et al. Computer-assisted path planning for percutaneous needle insertions, Medical Physics, 38(6):3246-59, 2011.

[8]Hamze N, Voirin J , Collet P , et al. Pareto front vs. Weighted sum for automatic path planning of deep brain stimulation. 2016.

[9]Baegert C, Villard C, Schreck P, Soler L. Multi-criteria path planning for hepatic radiofrequency ablation. Proceedings ofMICCAI, 2007.

[10] Craven B. D., Islam S. M. N., Mittnik S., et al. Optimization in Economics and Finance.dynamic modeling \& econometrics in economics \& finance, 7:xii, 2005. 